\title{
Effect of $\beta$-glucan molecular weight on rice flour dough rheology, quality parameters of breads and in vitro starch digestibility $^{*}$
}

Perez-Quirce, S., Lazaridou, A., Biliaderis, C.G., Ronda, F.

LWT - Journal of Food Science and Technology. (2017) Vol 82, pp: 446-453

DOI: 10.1016/j.lwt.2017.04.065. 


\title{
Effect of $\beta$-glucan molecular weight on rice flour dough rheology, quality parameters of breads and in vitro starch digestibility
}

\author{
Sandra Perez-Quirce ${ }^{1}$, Athina Lazaridou², Costas G. Biliaderis ${ }^{2}$, Felicidad \\ Ronda $^{1^{*}}$
}

${ }^{1}$ Department of Agriculture and Forestry Engineering, Food Technology, College of Agricultural and Forestry Engineering, University of Valladolid, Av. Madrid, 44, 34004 Palencia, Spain.

${ }^{2}$ Department of Food Science and Technology, School of Agriculture, Aristotle University of Thessaloniki, P.O. Box 235, Thessaloniki, Greece, 54124.

* Corresponding author: fronda@iaf.uva.es; Tel: +34979108339; Fax: $+34979108200$

\begin{abstract}
The study aimed at investigating the effects of molecular weight(peak molecular weight, Mp, 83, 192 and $650 \mathrm{kDa}$ ) and level (1.3, 2.6 and $3.9 \mathrm{~g} / 100 \mathrm{~g}$ flour basis) of enriched in $\beta$-glucan (BG) concentrates (from oat and barley) added into rice flour gluten-free (GF) doughs on their viscoelastic and pasting properties, as well as the quality parameters of bread and the in vitro starch digestibility. A purification process of a commercial BG concentrate, followed by an acid hydrolysis step were employed to reduce the content of interfering excipients (e.g. maltodextrins) and obtain preparations with a range of molecular weights. BG-enriched GF breads of improved quality, that can fulfil the EFSA claims (ingest of $3 \mathrm{~g}$ of BG per day with a daily bread intake of 200 g of bread), were obtained, exhibiting slower starch digestibility (in vitro assay) dependent on the molecular weight and concentration of BG. With the higher Mp BG used, showing the largest impact on dough rheology characteristics and having a greater potential for health benefits, higher specific volume and lower bread crumb hardness were noted among the GF breads. The medium and lowest Mp BG also had an influence on dough rheological behavior and bread quality attributes. The rapidly available glucose of the bread decreased from $81 \mathrm{~g} / 100 \mathrm{~g}$ to $72 \mathrm{~g} / 100 \mathrm{~g}$ as result of the $3.9 \mathrm{~g} / 100 \mathrm{~g}$ addition of the highest Mp BG in the GF formulations.
\end{abstract}


Keywords: Gluten free bread; pasting properties; oscillatory test, creep-recovery test; digestible starch.

\section{Introduction}

In recent years $\beta$-glucans (BG) have received significant consumer and research attention because their consumption has been linked with certain health benefits recognized by EFSA (EFSA 2011). These health claims have largely contributed to increased consumption of cereal based foods, such as breads, muffins, pasta and breakfast cereals, mostly whole flour products (Wolever et al., 2010). However, celiacs cannot ingest wheat, barley or even oat, the main sources of this dietary fiber, since they are not gluten-free grains. The BG enrichment of gluten-free breads to fulfil the requirements for the above EFSA claims can be achieved by addition of BG concentrates obtained from these cereals by means of water extraction processes that cannot co-solubilize the allergenic storage proteins of the prolamin fraction (PerezQuirce, Collar \& Ronda, 2014; Ronda, Perez-Quirce, Angioloni \& Collar, 2013; Ronda, Perez-Quirce, Lazaridou \& Biliaderis, 2015).

The demand for gluten-free (GF) products steadily increases as a result of enhanced consumer education on diet related pathogenic conditions. Despite the current demand for development of food products with improved nutritional quality, the GF products often receive only marginal attention from a nutrient-rich formulation point of view. The enrichment of GF breads with BG, does hold a special interest among the vulnerable population of celiac patients which also encounters a significant incidence rate of other associated chronic diseases, such as obesity-metabolic syndrome and diabetes due to their higher fat and calorie denser diets compared to the general population (Cronin \& Shanahan, 1997).

The functionality and physiological properties of cereal BG are strongly related to their viscosity, which depends on the molecular weight $(\mathrm{Mw})$, fine structure, concentration and physical state of the polysaccharide (Lazaridou \& Biliaderis, 2007; Wolever et al., 2010). Generally, high Mw BG are reported to enhance the viscosity of the liquid in the upper digestive tract (digesta) and, thereby, it is beneficial for exerting many physiological activities (Wood, 1994); it reduces the peak blood glucose response (Regand, 2009) and attenuates the glycemic response and the in vitro starch digestion (Thondre, Monro, Mishra \& Henry, 2010). Overall, the Mw 
and amount of BG solubilised in the gut might be critical in its capacity to reduce glucose release and transport as a function of the increased viscosity of the digesta and reduced motility (Regand, Chowdhury, Tosh, Wolever \& Wood, 2011).

Previous studies have shown a great variability in the effect of BG on dough viscoelastic behaviour and bread quality characteristics of wheat flour bakery items (Brennan \& Cleary, 2007; Cleary, Andersson \& Brennan, 2007; Skendi, Biliaderis, Papageorgiou \& Izydorczyk, 2010) and GF products (Hager et al., 2011; Lazaridou, Duta, Papageorgiou, Belc \& Biliaderis, 2007; Perez-Quirce, 2014; Ronda et al., 2013), depending on dough water and BG contents. A loss of quality when GF breads are fortified with BG is partially related to their $\mathrm{Mw}$, as a result of enhanced water-binding and viscosity (Perez-Quirce et al., 2014; Ronda et al., 2015). Thus, although the $\mathrm{Mw}$ is a key determinant of the health promoting potential of BG, addition of high levels of high Mw BG preparations can be a challenge for food producers because of their low solubility and high viscosity, affecting detrimentally the dough handling properties and the sensory attributes of the final product. These negative changes could be avoided by incorporating lower BG concentrations and/or BG preparations of lower Mw (Kennan et al., 2007). However, with lower Mw BG, a reduction in physiological responses is anticipated (Cleary et al., 2007).

The effects of Mw on GF dough and bread quality have mostly been studied by means of commercial BG concentrates, often of low purity. In this context, the BGenrichment is accompanied by addition of high amounts of other substances, such as maltodextrins, present in the commercial product $(\sim 72 \mathrm{~g} / 100 \mathrm{~g})$. Maltodextrins could have adverse effects, such as weakening of the dough network structure (Witczak, Korus, Ziobro \& Juszczak, 2010) or dough stickiness, and thereby modify the true effects caused by BG itself (Ronda et al., 2015). Moreover, maltodextrins, being starch hydrolysis products, can aggravate the glycemic responses of the final products since they are a form of readily digestible carbohydrates. Thus, a comprehensive study of the effects of Mw and amount of highly concentrated BG on GF dough and bread properties is required.

This study reports on the enrichment in BG of a commercial BG concentrate by means of a purification protocol and a reduction of their Mw by acid hydrolysis of the polysaccharides in a paste form of the enriched BG preparation. Under properly 
selected hydrolysis conditions the production of a tailor-made lower Mw BG preparation was feasible. Moreover, this study aimed at investigating the effects of Mw and level of the BG preparations introduced into rice flour GF doughs on their viscoelastic and pasting properties and the quality parameters of the resultant breads, bread volume and firmness. The impact of BG enrichment on the in vitro starch digestibility of breads was also assessed.

\section{Materials and methods}

\subsection{Materials}

Rice flour (12.5 g/100 g moisture, $0.46 \mathrm{~g} / 100 \mathrm{~g}$ ash, $7.5 \mathrm{~g} / 100 \mathrm{~g}$ protein, $0.49 \mathrm{~g} / 100 \mathrm{~g}$ fat and $79.1 \mathrm{~g} / 100 \mathrm{~g}$ starch) was supplied by Herba Ricemills S.L.U (Tarragona, Spain). Salt, sugar, and sunflower oil were purchased from the local market. Hydroxypropyl-methyl-cellulose (HPMC) 4KM was a gift from Dow Chemical (Midland, USA).

Barley $(1 \rightarrow 3)(1 \rightarrow 4)-\beta$-D-glucan (BBG)(Glucagel $\left.{ }^{\mathrm{TM}}\right)$, a low Mw BG, was given as a free sample from DKSH (Hamburg, Germany). The oat $(1 \rightarrow 3)(1 \rightarrow 4)-\beta$-D-glucan concentrate (OBG) (Promoat ${ }^{\mathrm{TM}}$ ) which was a high Mw BG preparation, was supplied by Biovelop AB (Kimstad, Sweden). The proximate composition of these materials as given by the suppliers was: for BBG, $2.52 \mathrm{~g} / 100 \mathrm{~g}$ moisture, $4.75 \mathrm{~g} / 100 \mathrm{~g}$ soluble protein, $1.43 \mathrm{~g} / 100 \mathrm{~g}$ ash, $1.32 \mathrm{~g} / 100 \mathrm{~g}$ fat, $>85 \mathrm{~g} / 100 \mathrm{~g}$ total carbohydrates, and $>72$ g/100 g BG; for OBG, 6 g/100 g moisture, 54-56 g/100 g carbohydrates (dextrin), $<4.5 \mathrm{~g} / 100 \mathrm{~g}$ protein, 1-3 g/100 g ash and 0.5-1 g/100 g fat, and 33-36 g/100 g BG. The gluten content of the commercial BBG and OBG samples was analyzed by the ELISA test based on the R5 antibody; for OBG, the gluten content was under the detection limit ( $<6.2 \mathrm{mg} / \mathrm{kg}$ ), while for BBG $1.76 \mathrm{~g} / \mathrm{kg}$, which rendered the latter BG concentrate as not gluten-free. Nevertheless, the BBG was included in this study as a raw material for evaluation of the effect of BG Mw on the rice flour-based glutenfree dough formulations since it is technically feasible to obtain a gluten-free barley BG concentrate (Ronda et al., 2013).

\section{2. $\beta$-glucan isolation and characterization}


The OBG was of relatively low purity in BG (33-36 g/100 g). Hence, for this study further purification of this preparation was carried out, by a modification of the method of Lazaridou, Biliaderis, Micha-Screttas \& Steele (2004) to obtain a preparation with much higher BG concentration ( $70 \mathrm{~g} / 100 \mathrm{~g})$ as shown in Figure 1; this concentrate was designated as HWB. Following the method of Sibakov et al. (2013) with some modifications, an amount of this enriched HWB concentrate was subsequently hydrolyzed by a $8 \mathrm{~g} / 100 \mathrm{~mL}$ phosphoric acid solution at $82{ }^{\circ} \mathrm{C}$ acting for 25 min to obtain a second BG preparation with a desired medium Mw (MWB). The hydrolysis step was carried out at high solids level (paste like mixture) to more effectively control the degradation rate, yielding the preferred $\mathrm{Mw}$ of the polysaccharide. The entire purification/isolation protocol is outlined in detail in Fig 1. The $\beta$-glucan content and the apparent peak molecular weight $(\mathrm{Mp})$ of the isolated BG preparations were determined using the mixed-linkage $(1 \rightarrow 3)(1 \rightarrow 4) \beta$-D-glucan assay kit purchased from Megazyme (Megazyme International Ireland Ltd., Co., Bray, Ireland) as well as a high performance size exclusion chromatography system with a refractive index detector, respectively. The running conditions of the chromatography and the sample preparation are described in detail elsewhere (Lazaridou et al., 2004; Lazaridou, Marinopoulou, Matsoukas \& Biliaderis, 2014). The low Mw BG (LWB) preparation used in this study for bread enrichment was the commercial product Glucagel ${ }^{\circledR}$ (BBG), having an adequate BG content (72 g/100 g) for the requirements of the present work. 


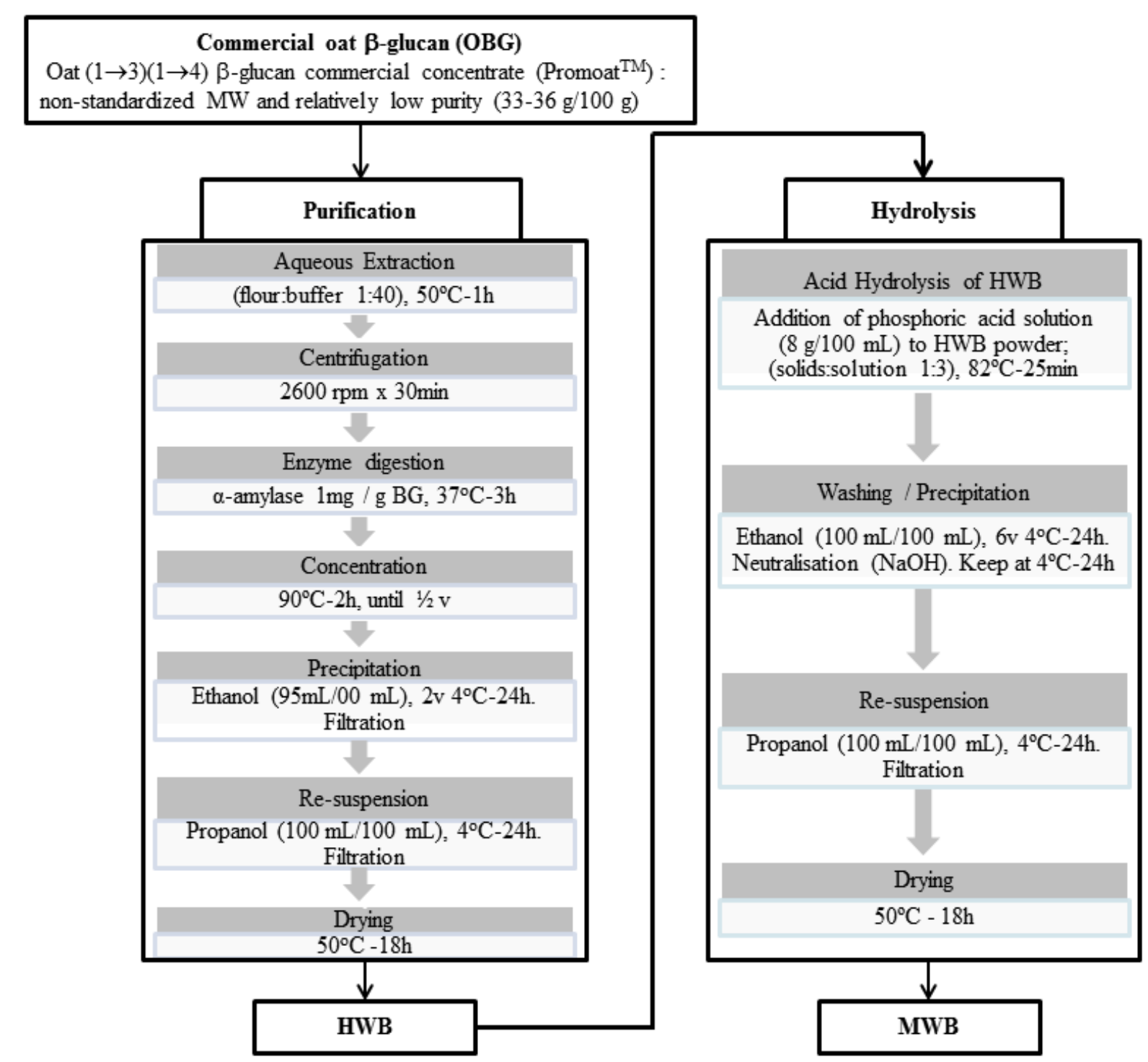

Figure 1: Flowchart of purification of the commercial $\beta$-glucan and hydrolysis process. MW: Molecular Weight; HWB: $\beta$-glucan of high molecular weight; MWB: $\beta$-glucan of medium molecular weight. 


\subsection{Dough preparation and breadmaking}

A straight dough process was performed for the breadmaking using the following ingredient formulation expressed on a $100 \mathrm{~g}$ rice flour basis: $6 \mathrm{~g}$ oil, $5 \mathrm{~g}$ sucrose, $2 \mathrm{~g}$ HPMC, $1.8 \mathrm{~g}$ salt and $3 \mathrm{~g}$ dried yeast. The levels of BG incorporated into this formulation were 0 (control), 1.3, 2.6 and $3.9 \mathrm{~g} / 100 \mathrm{~g}$ (rice flour basis) of pure BG by adding different amounts of BG preparations according to their BG contents and added on top of the other ingredients. The amounts of added water to the dough were adapted according to the BG level based on the findings of our previous work (Ronda et al., 2015) and were 92, 105, 120 and 130 g/100 g (rice flour basis) with increasing BG content from 0 to $3.9 \mathrm{~g} / 100 \mathrm{~g}$. Dough rheological tests were performed without inclusion of yeast in the formulated doughs in order to obtain stable readings (avoiding $\mathrm{CO}_{2}$ evolution). BG samples were hydrated in hot water (40-45 ${ }^{\circ} \mathrm{C}$ ) prior to their mixing with the remaining dough ingredients. The GF dough and breadmaking procedures are described in detail elsewhere (Ronda et al., 2015). After baking, breads were removed from the pan and stored for one hour at room temperature before any analysis.

\subsection{Evaluation of dough rheology}

\subsubsection{Fundamental rheology}

Oscillatory and creep-recovery tests were carried out at least in duplicate and triplicate, respectively with a RheoStress 1 rheometer (Thermo Haake, Karlsruhe, Germany) following the same procedure described in detail elsewhere (Ronda et al., 2013; 2015). A stress sweep from 0.1 to $200 \mathrm{~Pa}$ at $1 \mathrm{~Hz}$ and $25{ }^{\circ} \mathrm{C}$ was performed to establish the linear viscoelastic region (LVR). Frequency sweep data were fitted to a power law model as previously described by Ronda et al. (2013). The recorded viscoelastic parameters, $\mathrm{G}_{1}$ ' and $\mathrm{G}_{1}$ ', and $\tan \delta_{1}$, represent the elastic and viscous moduli and the loss tangent, respectively, at a frequency of $1 \mathrm{~Hz}$. The a, b and c exponents quantify the dependence of the dynamic moduli and the loss tangent on the oscillation frequency.

Creep tests were performed by imposing a step of shear stress of $50 \mathrm{~Pa}$, exceeding the LVR, for $60 \mathrm{~s}$. In the recovery phase, the stress was suddenly removed and the sample was allowed for $180 \mathrm{~s}$ to recover the elastic (instantaneous and retarded) part 
of the deformation. Creep data are described in terms of creep compliance, J, which is defined as the strain divided by the stress applied. The data from creep and recovery tests were modelled to the 4- and 3-parameter Burger's models, respectively (Lazaridou et al., 2007; Ronda et al., 2013). Concerning the calculated parameters, $\mathrm{J}_{0}$ is the instantaneous compliance, $\mathrm{J}_{1}$ is the retarded elastic or viscoelastic compliance, $\lambda_{1}$ is the retardation time and $\eta_{0}$ is the steady viscosity (estimated from the creep step). Additionally, $\mathrm{J}_{\max }$ is the maximum creep compliance obtained at the end of the creep step and $\mathrm{J}_{\text {steady }}$, the steady-state compliance in the recovery step, calculated by subtracting the compliance value at the terminal region of curve (where dough recovery reached equilibrium) from the $\mathrm{J}_{\max }$. Recovery (\%) was calculated as $100 \cdot\left(\mathrm{J}_{\text {steady }} / \mathrm{J}_{\max }\right)$.

\subsubsection{Pasting properties}

Viscometric profiles of formulated and then lyophilized rice flour doughs (without yeast) were obtained with a Rapid Visco Analyser (RVA-4, Newport Scientific, Warriewood, Australia) using the ICC Standard 162 protocol. The re-hydrated freeze-dried samples were transferred into RVA canisters and processed as previously described by Ronda et al. (2013). These tests were carried out in triplicate.

\subsection{Evaluation of bread quality}

Bread volume was determined in duplicate by a Volscan profiler 300 analyser (Stable Microsystems, Godalming, UK). Breads were weighed immediately after removal from the pan once cooled down. Crumb hardness was determined in duplicate with a TA-XT2 texture analyser (Stable Microsystems, Surrey, UK) using the software "Texture Expert”. An aluminum 20 mm diameter cylindrical probe was employed in a compression test at $1 \mathrm{~mm} / \mathrm{s}$ speed test and deformation level up to 50 \%. Crumb hardness $(\mathrm{N})$ was calculated from the maximum force of the curves. Analysis was carried out at $20 \pm 3{ }^{\circ} \mathrm{C}$ on bread slices, with $20 \mathrm{~mm}$ thickness, taken from the center of each loaf.

\subsection{In vitro starch digestibility of breads}

In vitro starch enzymatic digestibility of breads was measured according to the modified method of Englyst, Hudson \& Englyst (2000). The hydrolyzed glucose 
released by digestive enzymes at $20 \mathrm{~min}\left(\mathrm{G}_{20}\right)$ and $120 \mathrm{~min}\left(\mathrm{G}_{120}\right)$ and the total glucose (TG) content were determined by the glucose oxidase/peroxidase colorimetric method. The free sugar glucose (FGS) content was also determined through a separate test following the procedure proposed by Englyst et al. (2000). From these tests, rapidly digested starch (RDS), slowly digestible starch (SDS), resistant starch (RS), total starch (TS) and rapidly available glucose (RAG) were calculated. Starch digestibility rate index (SDRI) was computed from the percentage of RDS in TS of the breads. This test was carried out in quadruplicate.

\subsection{Statistical analyses}

Statgraphics Centurion v.16 (Bitstream, Cambridge, MN, USA) software was used for the non-linear regressions and for Pearson correlation analysis. STATISTICA package (Tulsa, OK, EEUU) v.6 was used for ANOVA analysis. Fisher's least

significant difference (LSD) test was adopted to evaluate significant differences $(\mathrm{p}<0.05)$ among samples. Homogeneity of variance was checked for each studied variable.

\section{Results and discussion}

The three BG preparations (LWB, MWB and HWB) employed in this study largely differed in molecular size, having peak molecular weight values of $83 \mathrm{kDa}, 192 \mathrm{kDa}$ and $650 \mathrm{kDa}$ (Fig. 2) and BG contents of 72 g/100 g, 68 g/100 g and 73 g/100 g, respectively.

\subsection{Fundamental rheology of bread doughs}

The effect of addition of enriched BG concentrates on rheological properties of GF doughs prepared with the optimum hydration level (to maximize the bread making performance of the composite doughs) was investigated in this study. The elastic modulus values (1439 $\mathrm{Pa}<\mathrm{G}_{1}<11187 \mathrm{~Pa}$ ) were higher than those of viscous modulus (690 $\mathrm{Pa}<\mathrm{G}^{\prime \prime}<3120 \mathrm{~Pa}$ ), and the values for $\tan \delta_{1}$ were $<1$ for the dough formulations (Table 1). Both moduli slightly increased with frequency; this dependence, which is quantified by the 'a' and 'b' exponents, calculated from G' and G' fittings to the power law model, became less pronounced with BG addition (Table 1). The visco- 
elastic behaviour of all doughs corresponded to solid-like materials, in agreement with earlier findings on GF doughs enriched with BG concentrates (Lazaridou et al., 2007; Ronda et al., 2015).

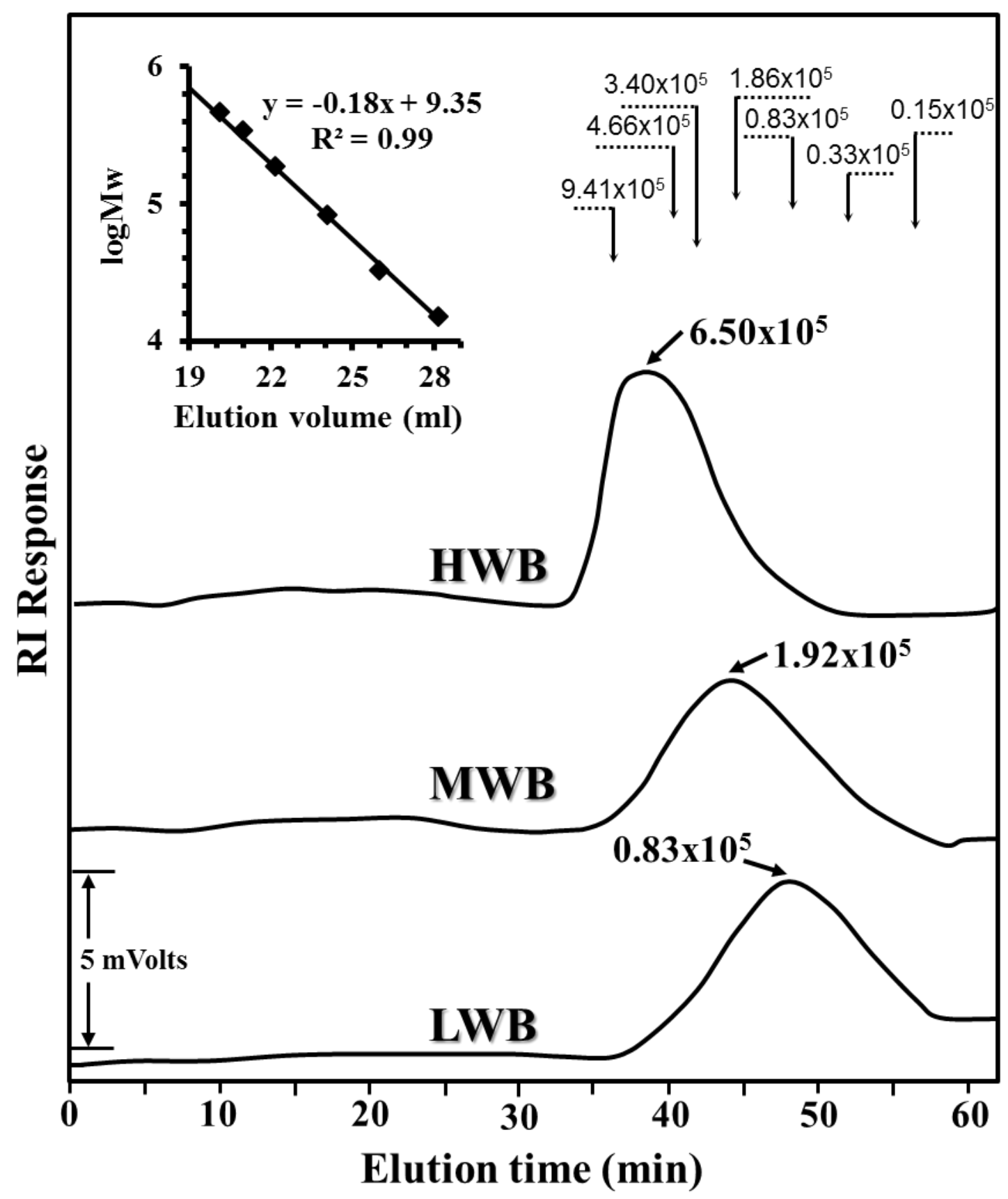

Figure 2. HPSEC elution profiles and peak molecular weight (Mp) (slanted arrows) of the eluting peaks of enriched in $\beta$-glucan concentrates added to the GF bread formulations, as detected by RI. The vertical arrows indicate the elution time of the peak fraction of seven $(1 \rightarrow 3)(1 \rightarrow 4)$ - $\beta$-D-glucan standards (Mp: 0.15, 0.33, 0.83, 1.86, 3.40, 4.66 and $9.41 \times 10^{5}$ ) used for plotting of the molecular weight-elution volume standard curve (inset). LWB: $\beta$-glucan of low molecular weight; MWB: $\beta$ glucan of medium molecular weight; HWB: $\beta$-glucan of high molecular weight. 
Table 1. Effects of molecular weight and level of added $\beta$-glucan on viscoelastic parameters of $\beta$-glucan-enriched rice flour-based (gluten-free) doughs and specific volume and crumb hardness of breads.

\begin{tabular}{|c|c|c|c|c|c|c|c|c|c|c|c|c|c|c|c|c|c|c|c|c|c|c|}
\hline \multirow{2}{*}{\multicolumn{2}{|c|}{$\begin{array}{l}\text { BG Mp } \\
\text { BG level (g/100 g flour basis) }\end{array}$}} & \multirow{2}{*}{\multicolumn{2}{|c|}{$\frac{\text { CONTROL }}{0}$}} & \multicolumn{6}{|c|}{ LWB } & \multicolumn{6}{|c|}{ MWB } & \multicolumn{6}{|c|}{ HWB } & \multirow{2}{*}{ SE } \\
\hline & & & & \multicolumn{2}{|c|}{1.3} & \multicolumn{2}{|c|}{2.6} & \multicolumn{2}{|c|}{3.9} & \multicolumn{2}{|c|}{1.3} & \multicolumn{2}{|c|}{2.6} & \multicolumn{2}{|c|}{3.9} & \multicolumn{2}{|c|}{1.3} & \multicolumn{2}{|c|}{2.6} & \multicolumn{2}{|c|}{3.9} & \\
\hline \multirow{6}{*}{ 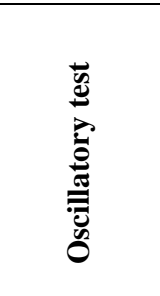 } & $\mathrm{G}_{1}^{\prime}(\mathrm{Pa})$ & 3361 & C & 3243 & c & 2465 & $\mathrm{~b}$ & 9745 & $\mathrm{e}$ & 1439 & $\mathrm{a}$ & 1729 & $\mathrm{a}$ & 3228 & $\mathrm{C}$ & 3128 & $\mathrm{bc}$ & 5936 & $\mathrm{~d}$ & 11187 & $\mathrm{f}$ & 250 \\
\hline & $\mathbf{a}$ & 0.283 & e & 0.279 & de & 0.282 & de & 0.167 & $\mathrm{a}$ & 0.276 & de & 0.245 & cd & 0.219 & bc & 0.308 & $\mathrm{e}$ & 0.227 & bc & 0.199 & $\mathrm{ab}$ & 0.016 \\
\hline & $\mathrm{G}^{\prime \prime}{ }_{1}(\mathrm{~Pa})$ & 1512 & d & 1418 & d & 1025 & c & 2436 & $\mathrm{f}$ & 702 & $\mathrm{ab}$ & 690 & $\mathrm{a}$ & 956 & bc & 1536 & $\mathrm{~d}$ & 2002 & e & 3120 & g & 106 \\
\hline & b & 0.337 & $\mathrm{f}$ & 0.306 & def & 0.333 & ef & 0.256 & $\mathrm{ab}$ & 0.293 & bcde & 0.278 & abcd & 0.251 & $\mathrm{a}$ & 0.322 & ef & 0.300 & cde & 0.268 & $\mathrm{abc}$ & 0.014 \\
\hline & $\tan \delta_{1}=G^{\prime \prime} / G^{\prime}$ & 0.449 & e & 0.437 & de & 0.417 & de & 0.250 & $\mathrm{a}$ & 0.488 & f & 0.403 & d & 0.297 & bc & 0.492 & $\mathrm{f}$ & 0.337 & c & 0.279 & $\mathrm{ab}$ & 0.016 \\
\hline & c & 0.054 & cd & 0.027 & abc & 0.051 & bcd & 0.089 & $\mathrm{e}$ & 0.017 & $\mathrm{ab}$ & 0.033 & abc & 0.032 & $\mathrm{abc}$ & 0.014 & a & 0.073 & de & 0.069 & de & 0.012 \\
\hline \multirow{9}{*}{ 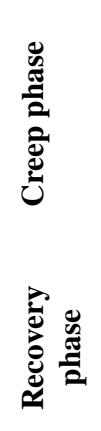 } & $\mathrm{J}_{0}\left(10^{-4} \mathrm{~Pa}^{-1}\right)$ & 2.2 & $\mathrm{ab}$ & 3.0 & c & 3.0 & c & 2.2 & $\mathrm{ab}$ & 4.0 & $\mathrm{~d}$ & 6.9 & $f$ & 6.0 & $\mathrm{e}$ & 1.7 & $\mathrm{a}$ & 2.7 & bc & 1.9 & $\mathrm{a}$ & 0.2 \\
\hline & $\mathrm{J}_{1}\left(10^{-4} \mathrm{~Pa}^{-1}\right)$ & 40.5 & e & 37.0 & de & 30.7 & c & 4.2 & $\mathrm{a}$ & 20.4 & $\mathrm{~b}$ & 40.1 & e & 18.8 & $\mathrm{~b}$ & 34.5 & $\mathrm{~cd}$ & 6.2 & a & 2.6 & $\mathrm{a}$ & 1.6 \\
\hline & $\lambda$ & 11.3 & e & 11.8 & e & 11.6 & e & 8.1 & bcd & 5.2 & a & 6.6 & abc & 9.1 & $\mathrm{~d}$ & 10.8 & e & 8.2 & bcd & 6.6 & $\mathrm{abc}$ & 0.6 \\
\hline & $\eta_{0}\left(10^{3} \mathrm{~Pa} \cdot \mathrm{s}\right)$ & 2.4 & a & 2.7 & a & 2.4 & a & 162.1 & $\mathrm{~d}$ & 1.0 & a & 1.4 & $\mathrm{a}$ & 37.3 & $\mathrm{~b}$ & 2.4 & a & 124.3 & c & 376.3 & e & 11 \\
\hline & $J_{\max }\left(10^{-4} \mathrm{~Pa}^{-1}\right)$ & 309 & bc & 254 & $\mathrm{~b}$ & 337 & c & 10 & $\mathrm{a}$ & 640 & e & 461 & d & 39 & $\mathrm{a}$ & 310 & bc & 14 & a & 6.2 & a & 23 \\
\hline & $\mathrm{J}_{0}\left(10^{-4} \mathrm{~Pa}^{-1}\right)$ & 4.7 & bc & 6.2 & c & 5.7 & c & 3.7 & $\mathrm{ab}$ & 4.2 & $\mathrm{abc}$ & 13.9 & e & 10.1 & $\mathrm{~d}$ & 4.9 & bc & 4.6 & abc & 2.8 & $\mathrm{a}$ & 0.7 \\
\hline & $\mathrm{J}_{1}\left(10^{-4} \mathrm{~Pa}^{-1}\right)$ & 19.3 & c & 21.3 & $\mathrm{~cd}$ & 18.2 & c & 1.9 & $\mathrm{a}$ & 37.3 & e & 37.7 & e & 10.1 & $\mathrm{~b}$ & 22.6 & $\mathrm{~d}$ & 3.7 & a & 1.6 & a & 1.6 \\
\hline & $\lambda$ & 14.7 & a & 19.0 & d & 16.8 & c & 28.38 & $\mathrm{f}$ & 16.6 & c & 18.26 & d & 15.4 & $\mathrm{ab}$ & 18.3 & d & 15.8 & bc & 20.8 & $\mathrm{e}$ & 0.5 \\
\hline & Recovery (\%) & 7.6 & $\mathrm{ab}$ & 10.3 & $\mathrm{~b}$ & 7.1 & $\mathrm{ab}$ & 56.0 & $\mathrm{~d}$ & 5.3 & $\mathrm{a}$ & 9.73 & $\mathrm{~b}$ & 50.9 & $\mathrm{C}$ & 7.9 & $\mathrm{ab}$ & 60.7 & e & 73.0 & $\mathrm{f}$ & 0.8 \\
\hline \multirow{7}{*}{ 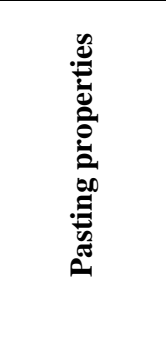 } & Peak viscosity (cp) & 998 & de & 800 & $\mathrm{~b}$ & 665 & $\mathrm{a}$ & 602 & $\mathrm{a}$ & 986 & de & 951 & cd & 789 & $\mathrm{~b}$ & 1080 & $\mathrm{f}$ & 912 & c & 1032 & ef & 21 \\
\hline & Trough viscosity (cp) & 833 & c & 659 & $\mathrm{~b}$ & 555 & a & 504 & a & 881 & c & 859 & c & 706 & $\mathrm{~b}$ & 992 & $\mathrm{~d}$ & 831 & c & 965 & $\mathrm{~d}$ & 19 \\
\hline & Breakdown (cp) & 165 & $\mathrm{f}$ & 142 & e & 110 & $\mathrm{~d}$ & 98 & bcd & 105 & $\mathrm{~cd}$ & 92 & bcd & 83 & $\mathrm{ab}$ & 88 & $a b c$ & 81 & $\mathrm{ab}$ & 67 & a & 7 \\
\hline & Final Viscosity(cp) & 1630 & c & 1182 & $\mathrm{~b}$ & 967 & a & 887 & a & 1799 & d & 1610 & c & 1257 & $\mathrm{~b}$ & 2107 & e & 1908 & d & 1874 & $\mathrm{~d}$ & 40 \\
\hline & Setback (cp) & 798 & c & 524 & $\mathrm{~b}$ & 413 & a & 383 & $\mathrm{a}$ & 919 & d & 751 & c & 552 & $\mathrm{~b}$ & 1115 & $\mathrm{e}$ & 1076 & e & 909 & $\mathrm{~d}$ & 33 \\
\hline & Peak Time (min) & 6.103 & a & 6.035 & $\mathrm{a}$ & 6.035 & a & 6.035 & $\mathrm{a}$ & 6.335 & $\mathrm{~b}$ & 6.365 & $\mathrm{~b}$ & 6.430 & bc & 6.535 & bc & 6.333 & $\mathrm{~b}$ & 6.665 & $\mathrm{C}$ & 0.085 \\
\hline & Pasting Temp. $\left({ }^{\circ} \mathrm{C}\right)$ & 88.0 & a & 90.5 & $\mathrm{~b}$ & 92.2 & bc & 92.7 & $\mathrm{C}$ & 91.8 & bc & 91.9 & bc & 92.7 & $\mathrm{C}$ & 92.3 & bc & 91.0 & bc & 93.1 & $\mathrm{C}$ & 0.67 \\
\hline \multirow{2}{*}{$\begin{array}{c}\text { Bread } \\
\text { properties }\end{array}$} & $\begin{array}{l}\text { Specific volume } \\
\text { (g/100mL) }\end{array}$ & 2.60 & c & 3.08 & e & 2.69 & $\mathrm{~d}$ & 2.04 & $\mathrm{a}$ & 3.03 & e & 2.70 & $\mathrm{~d}$ & 2.46 & $\mathrm{~b}$ & 3.17 & $\mathrm{f}$ & 2.63 & c & 2.60 & $\mathrm{C}$ & 0.019 \\
\hline & Hardness (N) & 2.38 & $\mathrm{~d}$ & 1.21 & $\mathrm{a}$ & 1.90 & bc & 6.53 & $\mathrm{f}$ & 1.37 & a & 1.67 & $\mathrm{ab}$ & 3.71 & $\mathrm{e}$ & 1.63 & $\mathrm{ab}$ & 2.63 & & 2.22 & $\mathrm{~cd}$ & 0.16 \\
\hline
\end{tabular}

BG: $\beta$-glucan; Mp: peak molecular weight; LWB: $\beta$-glucan of low molecular weight (83 kDa); MWB: $\beta$-glucan of medium molecular weight (192 kDa); HWB: $\beta$-glucan of high molecular weight $(650 \mathrm{kDa})$. The parameters from oscillatory tests correspond to the fitting of experimental measurements to power law model. The parameters from creep-recovery tests correspond to the fittings to Burger's model. Values in the same row with a letter in common are not significantly different $(\mathrm{p}>0.05)$. SE: Pooled standard error obtained from ANOVA analysis. Oscillatory tests and bread measurements were made at least in duplicate; creep-recovery tests were carried out at least in triplicate. 
Both viscoelastic moduli, G' and G", increased with BG addition to the doughs, with the extent of these changes being dependent on the Mp of BG, in accordance with the observations made by Cleary et al. (2007) and Ronda et al. (2015) for wheat-based, and GF rice flour-based doughs, respectively. The increments of moduli with respect to the control dough were larger for HWB, (averaged increasesof 201\%- and 147\% for $\mathrm{G}_{1}^{\prime}$ and $\mathrm{G}_{1}{ }_{1}$ respectively), than MWB increases of $63.4 \%$, and $51.8 \%$ for $\mathrm{G}_{1}^{\prime}$ and G"1 ) and LWB (increases of 153\%-, and 108\% for $\mathrm{G}_{1}^{\prime}$ and $\mathrm{G}_{1}{ }_{1}$-). This can be probably due to the large increase of water absorption capacity of the dough when high levels of high Mw BG preparations are included in the GF formulation (Ronda et al., 2015). Cleary et al. (2007) observed the same behavior in wheat doughs fortified with high Mw barley BG. According to the data, the $\mathrm{G}_{1}{ }^{\prime}$ and $\mathrm{G}_{1}$ " moduli of the MWB fortified doughs exhibited the lowest $G_{1}$ ' and $G_{1}$ " values among the three different BG samples. The barley LWB preparation indeed exhibits molecular features that could promote interchain (segmental) associations leading to gel formation due to its higher DP3 content (Lazaridou et al., 2004) and the much lower molecular weight compared to the oat BG preparations (MWB, HWB); formation of a gel network structure by barley BG could explain the higher consistency and strength of the respective doughs at an optimized hydration level compared to their MWB counterparts (Table 1). The enhancement of solid-like behavior of doughs with increased levels of oat BG concentrates is opposed to previous findings (Ronda et al., 2015) where similar tan $\delta_{1}$ values were reported for all levels of BG added to the GF doughs; this was attributed to a weakening effect of the maltodextrins present in the oat commercial BG concentrate (Witczak et al., 2010). The reduction of such excipients in the oat BG preparations of the present study could be responsible for the strengthening effect when the MWB and HWB were added to the GF doughs.

The obtained creep-recovery curves were similar to those previously found for rice flour and gluten-free doughs fortified with BG into the LVR (Ronda et al., 2013; 2015) or outside the LVR (Lazaridou \& Biliaderis, 2007). Both, the level and the Mp of the incorporated BG affected the creep-recovery parameters (Table 1). However, the effect on creep data was not proportional to the Mp of the added BG. High levels of LWB led to lower instantaneous $\left(\mathrm{J}_{0}\right)$ and retarded $\left(\mathrm{J}_{1}\right)$ elastic compliances $(\mathrm{p}<0.05)$ than those of MWB fortified doughs, associated to a lower deformation of the dough when submitted to a constant stress, probably due to the higher gelation 
capacity of the LWB preparation. These elastic compliances values were similar to those obtained for the HWB, which is probably attributed to its ability to form highly viscous (pseudoplastic) solutions (Lazaridou et al., 2004; Lazaridou \& Biliaderis, 2007).

Dough formulations with high elasticity, as manifested by low $\mathrm{J}_{0}$ and $\mathrm{J}_{1}$ compliances, could restrict dough expansion and lead to lower volume breads (Perez-Quirce et al., 2014). The elastic compliances of BG-formulated doughs although did not follow a clear trend with BG level, however, showed the lowest values for the highest levels of added BG preparations (Table 1). For the dough maximum compliance values, $\mathrm{J}_{\max }$, there was a decrease with increasing levels of added BG. A clear opposite trend was noted for the evolution of the steady state viscosity, $\eta_{0}$, which is measured from the reciprocal of flowability of the material at the end of the applied load in the creep test; it increased from $2.4 \mathrm{kPa} \cdot \mathrm{s}$ for the control dough to the $376 \mathrm{kPa} \cdot \mathrm{s}$ for the highest addition level of HWB, explaining the higher resistance to deformation under a constant stress of the latter sample; the former is in agreement with findings reported elsewhere, where high Mw $\beta$-glucans had significant higher viscosities in aqueous systems compared to their lower molar mass counterparts (Lazaridou et al., 2004; Mikkelsen et al., 2010). The recovery of the dough, when the stress was released, increased from $<10 \%$ for the control dough to $>70 \%$ for the HWB-fortified dough (Table 1). Taken into account that only the elastic part of the viscoelastic behavior can be recovered after application of a stress, this means that the $3.9 \mathrm{~g} / 100 \mathrm{~g}$ BGfortified doughs exhibited the most pronounced elastic behavior among all dough formulations. These data are consistent with the much lower $\tan \delta$ values observed by the oscillatory testing for all the 3.9g/100 g BG fortified doughs (Table 1).

\subsection{Pasting properties}

The impact of Mp and BG level on the RVA primary parameters is evidenced by the significant changes on the pasting and gelling behaviour of BG-enriched rice flourbased doughs (Table 1). Major single effects on cooking and cooling parameters were noted for the LWB GF formulation. With addition of LWB there was a significant $(\mathrm{p}<0.05)$ decrease of the peak (from $20 \%$ to $40 \%$ ), breakdown (from 14 $\%$ to $41 \%$ ), final (from $27 \%$ to $46 \%$ ) and setback (from $34 \%$ to $52 \%$ ) viscosities, compared to control, in accordance with findings reported for wheat flour substituted 
with 2.5 and 5 g/100 g BG (Brennan \& Cleary, 2007). A similar tendency was noted with incorporation of the MWB although this effect was significant at the highest level of substitution. A slight and opposite trend was observed in the case of HWB addition. Brennan \& Cleary (2007) proposed that barley BG could limit the available water within the paste mixture and hence limit the swelling of the starch granules, leading to greater retention of granular integrity and a reduction in gelatinization. A synergistic effect for the peak viscosity of rice starch and BG composite aqueous dispersions was reported by Banchathanakij \& Suphantharika (2009) and interpreted by assuming that the system is biphasic, with the BG located entirely in the continuous phase; as a result, its concentration increases as the volume of the phase accessible to the soluble hydrocolloid is reduced, due to swelling of the starch granules during pasting. The changes observed in the present study could be explained by a combination of these two phenomena, the water binding and the thickening of the continuous phase caused by BG addition; such opposing contributions to viscosity changes vary with the molecular weight and level of the soluble BG. It is also important to note that in the present work, compared to aforementioned studies, real dough systems were examined by RVA which consist of many flour ingredients, all contributing to the rheological responses.

The pasting temperature (PT) increased significantly with BG addition, regardless its Mp. The effect of fibres on the PT could be interpreted on the basis of the changes induced on granule swellingand amylose leaching processes responsible for commencement of starch pasting (Mira, Eliasson \& Persson, 2005). The observed decrease of breakdown with the inclusion of fibres denoted an increase in the paste resistance to cooking and a less intense shear thinning behavior; the latter implies less starch granule rupturing during cooking in the RVA. It was also noted that, the breakdown viscosity decreased with increasing Mp and BG fortification level.

The final viscosity (FV) decreased significantly, from $27 \%$ to $46 \%$ with LWB addition. Similarly, the setback viscosity decreased, up to $52 \%$ reduction (at 3.9 g/100 g added BG); such behavior can be attributed to a restriction of amylose retrogradation in the presence of LWB. However, the MWB and HWB oat BG showed different behavior; i.e., at the lowest level of added BG there was an increase in FV (10\% and $30 \%$ for MWB and HWB, respectively), while with further addition of BG lower FV values of the gelled BG-containing doughs were recorded. 
Similar responses were noted for the setback viscosities. In general, it is clear that with inclusion of high amounts of soluble dietary fibres in the composite mixtures, the intermolecular associations within the starch network upon cooling are weakened.

\subsection{Evaluation of bread quality}

The specific volume of breads was positively affected by the addition of BG at low levels; at $1.3 \mathrm{~g} / 100 \mathrm{~g}$ bread specific volume increased (17-22 \%) compared to the control bread, regardless the molecular weight of the BG preparation. However, fortification of the GF doughs with higher amounts of BG (up 3.9 g/100 g) reduced successively the loaf volume (Table 1). The lowest specific volume was presented for breads enriched with $3.9 \mathrm{~g} / 100 \mathrm{~g}$ LWB (Table 1 and Figure 3). Therefore, it seems that the structuring ability of the BG depends on its molecular weight and primarily on its concentration. The possible cause of the lowest specific volume of LWB could be the increased gelation potential of the BG that increases the rigidity of the doughs and thereby results in a lower dough expansion and loaf volume. The less pronounced loaf volume reduction with increasing contents of MWB and HWB preparations derived from the OBG concentrate could be attributed to the reduction and/or elimination of the maltodextrins in these samples; apparently, the addition of maltodextrins has a strong weakening effect on structure of GF dough and increases its susceptibility to deformation (Witczak et al., 2010). Other researchers have reported a significant decrease up to $50 \%$ in loaf volume and height with the inclusion of BG into wheat-based bread formulations (Brennan \& Cleary, 2007; Cleary et al., 2007; Hager et al., 2011); such a decline was more pronounced at higher levels of fortification. Skendi et al. (2010) demonstrated a dependence of loaf volume on the molecular size and concentration of barley BG added to the dough, as well as on the quality of wheat flour base used in the formulations. Other authors obtained the greatest reduction in loaf volume when a high Mw BG was added to wheat flour doughs (Cleary et al., 2007). Brennan \& Cleary (2007) observed that enrichment of dough with soluble BG preparations imparts reduced extensibility, which would limit oven spring. BG added to wheat doughs can interrupt the continuity of the gluten network (Skendi et al., 2010). In our case, the water amount added to the GF doughs was adjusted in such a way that BG enriched doughs showed neither the highest consistency nor the lowest compliances. The specific volume was 
correlated positively $(\mathrm{p}<0.001)$ to $\tan \delta_{1}$ values $(\mathrm{r}=0.91)$ and to the exponents a $(\mathrm{r}=0.91)$ and $\mathrm{b}(\mathrm{p}<0.05, \mathrm{r}=0.69)$ as well as negatively to $\mathrm{c}(\mathrm{p}<0.001 ; \mathrm{r}=-0.81)$ and the percentage of compliance recovery $(\mathrm{p}<0.05 ; \mathrm{r}=-0.70)$. The above relations mean that the higher the viscous-like behavior of the GF dough, with a structure more dependent on frequency, and the lower the ratio of elastic to total deformation under an applied stress, the higher the specific volume of the bread, at least within the range of values obtained for these variables in the current work.

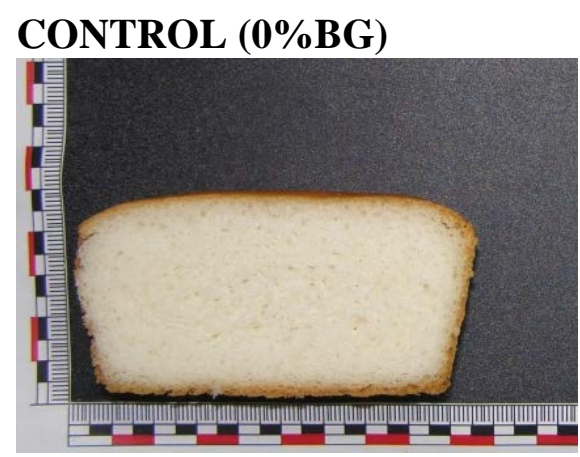

\section{9\% MWB}

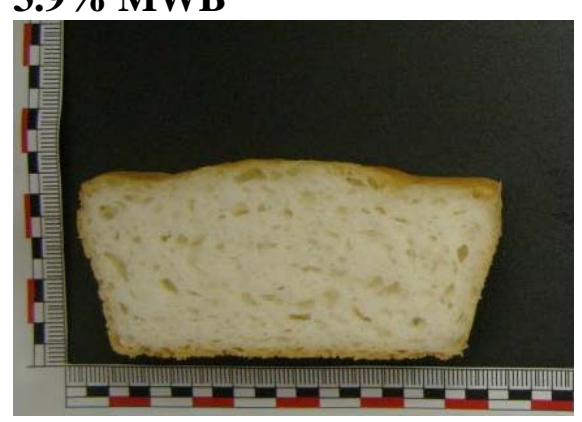

\section{$3.9 \%$ LWB}

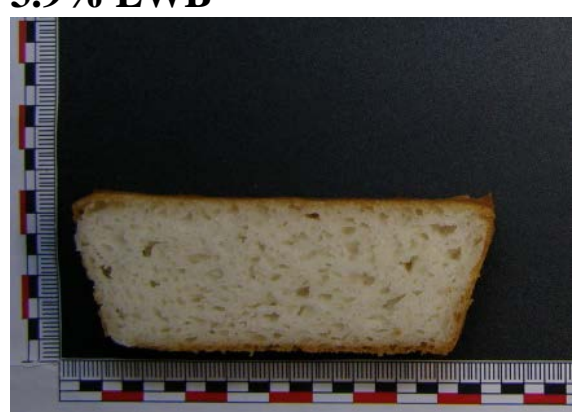

\section{$3.9 \%$ HWB}

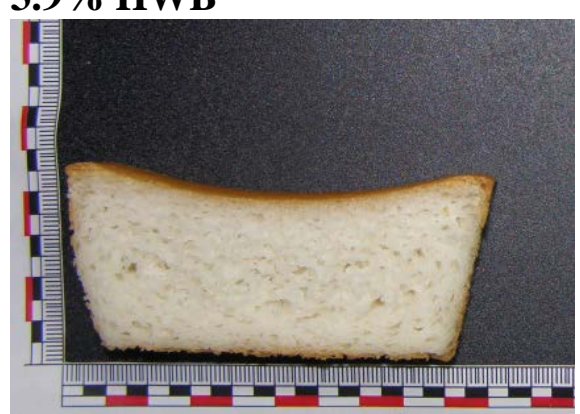

Figure 3. Cross-sections of gluten-free breads formulated with $\beta$-glucan preparations at the maximum level tested (3.9 g/100 g flour). BG: $\beta$-glucan; Mw: Molecular Weight; LWB: $\beta$-glucan of low molecular weight (83 kDa); MWB: $\beta$-glucan of medium molecular weight (192 kDa); HWB: $\beta$-glucan of high molecular weight (650 $\mathrm{kDa})$.

Enriched breads at the highest BG content showed the highest crumb hardness (Table 1); the highest value among the three different BG preparations was noted for the LWB samples, probably due to the lower loaf volume and the greater gelling potential of the LWB; correlation analysis showed that crumb hardness was negatively correlated with specific volume $(\mathrm{p}<0.001$; $\mathrm{r}=-0.89)$. A higher bread volume usually corresponds to higher amounts of air retained in the dough structure 
during proofing and baking, and this generally yields lower crumb hardness. Hager et al. (2011) also found a softening effect on the crumb with the incorporation of an oat BG preparation. Crumb hardness was further correlated (negatively) with the exponent a $(\mathrm{p}<0.01 ; \mathrm{r}=-0.80)$ and the $\tan \delta_{1}(\mathrm{p}<0.01 ; \mathrm{r}=-0.77)$ values of the dough, and positively correlated with the $c(p<0.05 ; \mathrm{r}=0.69)$ parameter of the dough

\subsection{In vitro starch digestibility of breads}

Table 2 shows the effect of inclusion of the three BG preparations at the lowest and highest concentration studied on the in vitro starch digestibility of the GF rice breads. Free sugar glucose (FSG) contents (g/100 g dry basis) of breads enriched with the lowest Mp BG were significantly lower $(\mathrm{p}<0.05)$ among all breads analyzed. Taken into account that sugar was added to the dough formulation at $5 \mathrm{~g} / 100 \mathrm{~g}$ level (rice flour basis) the low final concentration ( $<1 \mathrm{~g} / 100 \mathrm{~g}$ ) found in breads means that sucrose was largely used by yeast during fermentation. Rapidly available glucose (RAG) and rapidly digestible starch (RDS) values for breads with the lowest concentration of BG (1.3 g/100 g) were not different from the control regardless of the BG molecular weight. However, with increase of BG concentration there was a significant decrease of rapidly released glucose from starch by the digestive enzymes for MWB and HWB fortified breads, in agreement with previous in vivo studies (Stamataki et al., 2016). For RAG and RDS, the lowest values were achieved at the highest concentration of HWB, 72 g/100 g and 64 g/100 g versus 81 g/100 g and 72 $\mathrm{g} / 100 \mathrm{~g}$ in the control bread, respectively (Table 2), in agreement with a previous study (Hager et al., 2011). The digestible starch (DS) showed a small decrease in the presence of BG, yet large in cases of LWB and HWB inclusion at $3.9 \mathrm{~g} / 100 \mathrm{~g}$ level. As for the resistant starch (RS), at the highest fortification levels of BG, regardless of $\mathrm{Mp}$, there was a significant increase. The starch digestibility rate index (SDRI), which quantifies the starch digestion rate irrespective of the bread total starch content, showed a significant decrease in breads fortified with MWB and HMW preparations when the highest BG levels were used; this effect was enhanced with the increase of molecular weight of the BG preparation; at $3.9 \mathrm{~g} / 100 \mathrm{~g}$ addition of HWB the SDRI decreased from $93 \%$ (control) to $85 \%$. These observations related to the reduced rate and extent of starch digestion in the in vitro starch digestibility assays can be attributed to viscosity effects, as modulated by the concentration and 
molecular weight of the $\mathrm{BG}$ preparation ( $\mathrm{C} \times \mathrm{Mw}$ ); such trends have been also reported by Regand et al. (2011) for similar food products.

Table 2. Starch fractions FSG and RAG expressed as $\mathrm{g} / 100 \mathrm{~g}$ of total solids of the gluten-free breads (in vitro digestion).

\begin{tabular}{|c|c|c|c|c|c|c|c|c|}
\hline \multirow{2}{*}{$\begin{array}{l}\text { BG Mp } \\
\text { BG level } \\
\text { (g/100 g flour } \\
\text { basis) }\end{array}$} & \multirow{2}{*}{$\frac{\text { CONTROL }}{0}$} & \multicolumn{2}{|c|}{ LWB } & \multicolumn{2}{|c|}{ MWB } & \multicolumn{2}{|c|}{ HWB } & \multirow[b]{2}{*}{ SE } \\
\hline & & 1.3 & 3.9 & 1.3 & 3.9 & 1.3 & 3.9 & \\
\hline FSG & $0.84 \mathrm{~b}$ & 0.32 a & 0.38 a & $0.96 \mathrm{~b}$ & $0.60 \mathrm{ab}$ & $0.93 \mathrm{~b}$ & $0.96 \mathrm{~b}$ & 0.2 \\
\hline RAG & 80.5 с & 79.5 с & 78.0 bc & 80.0 с & 75.5 b & 77.7 bc & 71.5 a & 1.4 \\
\hline RDS & 71.7 c & 71.3 с & 69.8 bc & 71.2 с & $67.4 \mathrm{~b}$ & 69.1 bc & 63.5 a & 1.3 \\
\hline SDS & $4.9 \mathrm{~b}$ & $2.4 \mathrm{a}$ & $1.8 \mathrm{a}$ & $3.8 \mathrm{ab}$ & $7.6 \mathrm{c}$ & $4.9 \mathrm{~b}$ & $8.6 \mathrm{c}$ & 1.2 \\
\hline DS & 76.5 с & $73.7 \mathrm{ab}$ & 71.6 а & 75.0 bc & 75.0 bc & $74.4 \mathrm{~b}$ & $72.0 \mathrm{a}$ & 1.3 \\
\hline RS & $0.0 \mathrm{a}$ & $1.4 \mathrm{abc}$ & $4.6 \mathrm{~d}$ & $0.6 \mathrm{ab}$ & $1.6 \mathrm{bc}$ & $2.0 \mathrm{bc}$ & $2.9 \mathrm{~cd}$ & 1.0 \\
\hline TS & $76.3 \mathrm{ab}$ & 75.1 ab & $76.2 \mathrm{ab}$ & $75.6 \mathrm{ab}$ & 76.6 b & $76.1 \mathrm{ab}$ & 74.9 a & 1.0 \\
\hline SDRI & $93.3 \mathrm{c}$ & 94.8 с & 91.6 bc & 95.5 с & $88.0 \mathrm{ab}$ & 91.4 bc & 85.0 a & 0.02 \\
\hline
\end{tabular}

BG: $\beta$-glucan; Mp: peak molecular weight; LWB: $\beta$-glucan of low molecular weight (83 $\mathrm{kDa})$; MWB: $\beta$-glucan of medium molecular weight (192 kDa); HWB: $\beta$-glucan of high molecular weight $(650 \mathrm{kDa})$. FSG: Free glucose and sucrose; RAG = rapidly available glucose; RDS = rapidly digestible starch; SDS = slowly digestible starch; DS = digestible starch; RS = resistant starch; TS = total starch; and SDRI = starch digestion rate index (100 RDS/TS). SE: Pooled standard error from ANOVA analysis. Values with a letter in common in the same column are not significantly different $(\mathrm{p}>0.05)$. The tests were made at least in quadruplicate.

\section{Conclusions}

This study examined the potential impact of three different BG samples varying in their molecular weight and origin (oat and barley), but of similar purity, on physical and nutritional quality of rice flour based doughs and breads (gluten-free). A purification process of a commercial BG concentrate was adopted for the fortification of dough formulations to avoid the interference of other excipients present in this preparation and make clear the true impact of the BG addition in the composite formulation. Furthermore, it allowed the production of BG samples of reduced molecular weight by controlled acid hydrolysis of BG in a concentrated aqueous paste form. This study demonstrates the feasibility for production of BGenriched GF breads with acceptable quality attributes that can fulfill the EFSA claim 
(cholesterol lowering effect for daily consumption of $3 \mathrm{~g}$ BG with $\sim 200 \mathrm{~g}$ of fortified bread). It also indicated that formulation optimization (molecular weight and concentration of BG, as well as dough water content) is required to properly target for specific quality attributes of the end-product, while ensuring the full health potential of this physiologically active polysaccharide. Moreover, the use of high molecular weight BG preparations in bakery items must be preferred as it is well documented the relation between viscosity enhancement and hypoglycemic and hypocholesterolemic effects. Apparently, in the present study, at maximum fortification level (to justify the health claim requirements) acceptable quality characteristics (crumb texture) were noted with the highest molecular weight preparation. Finally, the results of this work can find special interest among the celiac patients who usually encounter great difficulty to access functional products, especially gluten free bakery items, with enhanced health benefits and acceptable sensory properties.

\section{Acknowledgement}

The authors gratefully acknowledge the financial support of the Spanish Institutions Ministerio de Economía y Competitividad and the European Regional Development Fund (FEDER) (Projects AGL2012-35088 and AGL2015-63849-C2-2-R).

\section{References}

Banchathanakij, R. \& Suphantharika, M. (2009). Effect of different $\beta$-glucans on the gelatinisation and retrogradation of rice starch. Food Chemistry, 114 (1), 5-14.

Brennan, C. S. \& Cleary, L. J. (2007). Utilisation of glucagel in the beta-glucan enrichment of breads: a physicochemical and nutritional evaluation. Food Research International, 40, 291-296.

Cleary, L. J., Andersson, R. \& Brennan, C. S. (2007). The behaviour and susceptibility to degradation of high and low molecular weight barley b-glucan in wheat bread during baking and in vitro digestion. Food Chemistry, 102, 889-897.

Cronin, C. \& Shanahan, F. (1997). Insulin-dependent diabetes mellitus and celiac disease. Lancet, 349(9058), 1096-1097. 
EFSA. (2011). Scientific Opinion on the substantiation of health claims related to beta-glucans from oats and barley and maintenance of normal blood LDLcholesterol concentrations (ID 1236, 1299), increase in satiety leading to a reduction in energy intake (ID 851, 852), reduction of post-prandial glycaemic responses (ID 821, 824), and “digestive function” (ID 850) pursuant to Article 13(1) of Regulation (EC) No 1924/2006. EFSA Journal, 9 (2207).

Englyst, K.N., Hudson, G.J., \& Englyst, H.N. (2000). Starch analysis in food. In: Meyers, R.A. (Ed.), Encyclopedia of Analytical Chemistry. John Wiley \& Sons, Chichester, pp. 4246-4262.

Hager, A. S., Ryan, L. A. M., Schwab, C., Ganzle, M. G., O'Doherty, J. V., \& Arendt, E. K. (2011). Influence of the soluble fibres inulin and oat beta-glucan on quality of dough and bread. European Food Research and Technology, 232(3), 405-413.

Keenan, J.M., Goulson, M., Shamliyan, T., Knutson, N., Kolberg, L., \& Curry, L., (2007). The effects of concentrated barley b-glucan on blood lipids in a population of hypercholesterolaemic men and women. British Journal of Nutrition, 97, 1162-1168.

Lazaridou, A. \& Biliaderis, C. G. (2007). Molecular aspects of cereal b-glucan functionality: physical properties, technological applications and physiological effects. Journal of Cereal Science, 46, 101-118.

Lazaridou, A., Biliaderis, C. G., Micha-Screttas, M., \& Steele, B. R. (2004). A comparative study on structure-function relations of mixed linkage (1/3), (1/4) linear b-D-glucans. Food Hydrocolloids, 18, 837-855.

Lazaridou, A., Duta, D., Papageorgiou, M., Belc, N., \& Biliaderis, C. G. (2007). Effects of hydrocolloids on dough rheology and bread quality parameters in gluten-free formulations. Journal of Food Engineering, 79(3), 1033-1047.

Lazaridou, A., Marinopoulou, A., Matsoukas, N. P., \& Biliaderis, C. G. (2014). Impact of flour particle size and autoclaving on b-glucan physicochemical properties and starch digestibility of barley rusks as assessed by in vitro assays. Bioactive Carbohydrates and Dietary Fiber, 4, 58-73.

Mikkelsen, M.S., Jespersen, B.M., Moller, B.L., Laerke, H.N., Larsen, F.H., \& Engelsen, S.B. (2010). Comparative spectroscopic and rheological studies on crude and purified soluble barley and oat beta-glucan preparations. Food Research International, 43 (10), 2417-2424.

Mira, I., Eliasson, A., \& Persson, K. (2005). Effect of surfactant structure on the pasting properties of wheat flour and starch suspensions. Cereal Chemistry, 82(1), 44-52

Perez-Quirce, S., Collar, C., \& Ronda, F. (2014). Significance of healthy viscous dietary fibres on the performance of gluten-free rice-based formulated breads. International Journal of Food Science and Technology, 49, 1375-1382. 
Regand, A. T. (2009). Physicochemical properties of beta-glucan in differently processed oat foods influence glycemic response. Journal of Agricultural and Food Chemistry, 57(19), 8831-8838.

Regand, A., Chowdhury, Z., Tosh, S.M., Wolever, T.M.S., \& Wood, P. (2011).The molecular weight, solubility and viscosity of oat beta-glucan affect human glycemic response by modifying starch digestibility. Food Chemistry, 129, 297304.

Ronda, F., Perez-Quirce, S., Angioloni, A., \& Collar, C. (2013). Impact of viscous dietary fibres on the viscoelastic behaviour of gluten-free formulated rice doughs: a fundamental and empirical rheological approach. Food Hydrocolloids, 32, 252262.

Ronda, F., Perez-Quirce, S., Lazaridou, A., \& Biliaderis C.G. (2015). Effect of barley and oat b-glucan concentrates on gluten-free rice based doughs and bread characteristics. Food Hydrocolloids, 48, 197-207.

Sibakov, J., Myllymäki, O., Suortti, T., Kaukovirta-Norja, A., Lehtinen, P., \& Poutanen K. (2013). Comparison of acid and enzymatic hydrolyses of oat bran $\beta$ glucan at low water content. Food Research International, 52, 99-108.

Skendi, A., Biliaderis, C. G., Papageorgiou, M., \& Izydorczyk, M. S. (2010). Effects of two barley b-glucan isolates on wheat flour dough and bread properties. Food Chemistry, 119, 1159-1167.

Stamataki, N. S., Yanni, A. E., Stoupaki, M. G., Tsigkas, A., Konstantopoulos, P., Pateras, I., Tentolouris, N., Perrea, D. N., \& Karathanos, V. T. (2016). Incorporation of beta-glucans into bread: postprandial glucose, insulin and ghrelin response of healthy subjects. Clinical Nutrition ESPEN. 13, e72, 2016.

Thondre, P.S., Monro, J.A., Mishra, S., \& Henry, C.J.K. (2010). High molecular weight barley b-glucan decreases particle breakdown in chapattis (Indian flat breads) during in vitro digestion. Food Research International, 43, 1476-1481.

Witczak, M., Korus, J., Ziobro, R., \& Juszczak, L. (2010). The effects of maltodextrins on gluten-free dough and quality of bread. Journal of Food Engineering, 96, 258-265.

Wolever, T.M.S., Tosh, S.M., Gibbs, A.L., Brand-Miller, J., Duncan, A.M., Hart, V., Lamarche, , Thomson, B.A. Duss, R., \& Wood, P.J. (2010). Physicochemical properties of oat $\beta$-glucan influence its ability to reduce serum LDL cholesterol in humans: a randomized clinical trial. American Journal of Clinical. Nutrition. 92, 723-32.

Wood, P. B. (1994). Effect of dose and modification of viscous properties of oat gum on plasma glucose and insulin following an oral glucose load. The British Journal of Nutrition, 72(5), 731-743. 

